\title{
Técnica inhalatoria en lactantes hospitalizados
}

\author{
YANAHARA SOLÍS O. ${ }^{1}$, GONZALO MENCHACA O., ${ }^{2,4}$, LUIS VEGA-BRICEÑO ${ }^{3,4}$, JAIME CERDA L. ${ }^{5}$ \\ 1. Programa de Especialidad en Pediatría. Facultad de Medicina, Pontificia Universidad Católica de Chile. \\ 2. Pediatra. Servicio Médico-Quirúrgico Infantil, Hospital Josefina Martínez (SSMSO). \\ 3. Pediatra Broncopulmonar. Hospital Josefina Martínez (SSMSO). \\ 4. Departamento de Pediatría. Pontificia Universidad Católica de Chile. \\ 5. Programa de Especialidad en Salud Pública. Facultad de Medicina, Pontificia Universidad Católica de Chile.
}

\begin{abstract}
Evaluation of the inhalatory technique in hospitalized infants

Background: The quality of the inhalatory technique (IT) is a factor on which its therapeutic success relies. Objective: Evaluate the quality of the IT performed by mothers of hospitalized infants due to acute respiratory infections, identifying possible mistakes. Method: 10 steps of an optimal IT were evaluated through a numerical scale previously designed, each step qualified with score 0 or 1 according to its accomplishment (maximum 10 points). The IT of each mother was evaluated and the correct accomplishment percentage of each step was calculated. Results: Mothers' IT scores presented a range of 4 to 10 points, where $12.5 \%$ reached the maximum score. The steps with the least accomplishment percentages were: appropriate waiting time between 2 inhalations (17.2\%), performance of a second inhalation (83.6\%) and holding the mouth and nose inside the inhalatory chamber for 10 seconds or 10 respiratory cycles once the inhalation had been administrated (89.1\%). Conclusion: Although the majority of mothers had received IT instruction and are familiarized with it, certain steps are still performed in a deficient way, compromising the therapy success.
\end{abstract}

(Key words: inhalation, evaluation studies, children).

Rev Chil Pediatr 2008; 79 (2): 152-156

\section{RESUMEN}

La calidad con que se realiza la técnica inhalatoria (TI) es un factor condicionante de su éxito terapéutico. Objetivo: Evaluar la calidad de la TI realizada por madres de lactantes hospitalizados por infecciones respiratorias agudas, identificando posibles errores. Pacientes y Métodos: Mediante una escala numérica previamente diseñada que incluyó 10 pasos de una TI óptima (calificada con puntaje 0 ó 1 según cumplimiento, puntaje máximo 10), se evaluó la TI de cada madre y el porcentaje de cumplimiento de cada paso. Resultados: El rango de calificaciones de las madres fue 4 a 10 puntos, obteniendo $12,5 \%$ el puntaje máximo. Los pasos que obtuvieron los menores porcentajes de cumplimiento fueron: tiempo de espera entre dos ciclos inhalatorios $(17,2 \%)$, realización de un segundo ciclo inhalatorio $(83,6 \%)$ y mantenimiento por 10 segundos o 10 respiraciones de la boca y nariz del lactante dentro de la aerocámara una vez

Trabajo recibido el 06 de agosto de 2007, devuelto para corregir el 24 de octubre de 2007, segunda versión el 27 de enero de 2008, aceptado para publicación el 26 de marzo de 2008.

Correspondencia a:

Jaime Cerda L.

E-mail: jcerda@med.puc.cl 
administrada la inhalación (89,1\%). Conclusión: A pesar de que la mayoría de las madres ha recibido instrucción y están familiarizadas con el uso de inhaladores, algunos pasos son realizados en forma deficiente por un elevado número de ellas, comprometiendo el éxito de la terapia.

(Palabras clave: aerosolterapia, técnica, evaluación, lactantes).

Rev Chil Pediatr 2008; 79 (2): 152-156

\section{Introducción}

El éxito de la terapia inhalatoria (aerosolterapia) depende de la calidad con que sea realizada, siendo la técnica inhalatoria (TI) probablemente más importante que las características del fármaco administrado, el sistema de inhalación, la edad del paciente y su condición respiratoria ${ }^{1}$. La instrucción de la TI tanto a pacientes como a sus cuidadores se realiza de manera verbal o demostrativa, considerándose necesario su reforzamiento periódico como garante de un cumplimiento adecuado. Esta tarea recae principalmente en médicos, kinesiólogos y personal de enfermería, tanto en escenarios ambulatorios como hospitalarios ${ }^{2-4}$. Numerosos estudios internacionales realizados en escolares y adultos han evaluado la calidad de la TI empleando diferentes metodologías de instrucción, demostrando la magnitud del efecto de una correcta educación sobre los pacientes y sus cuidadores ${ }^{5-8}$. Lamentablemente, muchos de sus hallazgos no son extrapolables a los niños menores de dos años, por cuanto actúan como sujetos pasivos al momento de ser inhalados; mas aún, probablemente los resultados publicados internacionalmente no reflejan nuestra realidad nacional, considerando las diferencias socioeconómicas y culturales existentes entre los países, las oportunidades de acceso a atención médica y los esfuerzos destinados a educación en salud. El presente estudio tiene por objetivo evaluar la calidad de la TI realizada por un grupo de madres de lactantes hospitalizados, identificando sus errores más frecuentes, sugiriendo finalmente algunas medidas destinadas a mejorar su desempeño.

\section{Pacientes y Métodos}

Durante los meses de julio y agosto del año 2006, se condujo un estudio descriptivo de corte transversal que evaluó la TI realizada por 128 madres de lactantes hospitalizados por infecciones respiratorias agudas (IRA) en el Servicio Médico-Quirúrgico Infantil del Hospital Josefina Martínez (SSMSO). Se obtuvo consentimiento informado verbal por parte de las madres, registrándose su edad y la edad de su hijo (a), nivel educacional según el último año cursado (básica, media o superior; esta última incluye cualquier tipo de estudio realizado tras finalizar cuarto año de enseñanza media), experiencia materna en la utilización de inhaladores en episodios obstructivos previos y tipo de instrucción recibida sobre TI (ninguna, verbal o demostrativa). Debido a que cada inhalador y aerocámara poseen un diseño único y, por consiguiente, diferentes instrucciones operativas para garantizar su utilización más adecuada ${ }^{1}$, la TI fue evaluada mediante una escala numérica diseñada por los autores que incluyó 10 pasos secuenciales (tabla 1). Cada paso se calificó con puntaje de 0 ó 1 de acuerdo a su cumplimiento, siendo el puntaje máximo de la escala 10 puntos. Se solicitó a cada madre realizar la TI en sus hijos verbalizando previamente sus acciones en cada paso, siendo calificadas por

\section{Tabla 1. Diez pasos evaluados durante la} realización de la técnica inhalatoria

\begin{tabular}{|c|c|}
\hline Paso $n^{\circ}$ & Acción realizada por la madre \\
\hline 1 & Agita el inhalador \\
\hline 2 & Inserta el inhalador en la aerocámara \\
\hline 3 & Posiciona correctamente al lactante \\
\hline 4 & $\begin{array}{l}\text { Posiciona correctamente la aerocámara sobre } \\
\text { boca y nariz }\end{array}$ \\
\hline 5 & Administra un sólo puff \\
\hline 6 & $\begin{array}{l}\text { Espera } 10 \text { segundos o } 10 \text { respiraciones del } \\
\text { lactante }\end{array}$ \\
\hline 7 & Remueve la aerocámara \\
\hline 8 & $\begin{array}{l}\text { Espera 30-60 segundos antes de repetir el } \\
\text { ciclo }\end{array}$ \\
\hline 9 & Agita nuevamente el inhalador \\
\hline 10 & Repite el ciclo completo \\
\hline
\end{tabular}




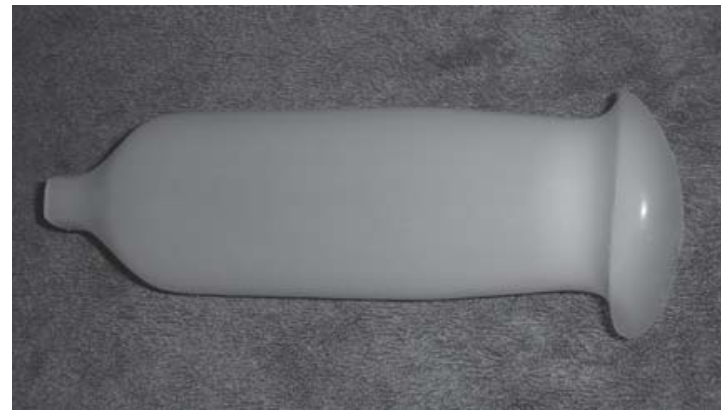

Figura 1. Aerocámara sin válvulas utilizada por las madres en la evaluación de la técnica inhalatoria.

un pediatra debidamente entrenado (YSO). Todas las madres utilizaron el mismo modelo de inhalador y aerocámara sin válvulas (figura 1) y sus errores fueron corregidos al término de cada evaluación. El análisis estadístico contempló el cálculo del puntaje obtenido por cada madre y el porcentaje de cumplimiento de cada uno de los pasos que componen la TI. Los datos fueron tabulados y analizados utilizando el programa estadístico minitab versión 14®.

\section{Resultados}

Madres y pacientes. La edad promedio de las madres fue 25 años (rango 16-45 años). De ellas, 113 (88\%) tenían experiencia con el uso de inhaladores en episodios obstructivos previos de IRA y 121 (95\%) contaban con instrucción previa de TI, 52 (41\%) en forma verbal y 69 (54\%) en forma demostrativa. Treinta y cuatro madres (27\%) habían cursado su último año de educación en la enseñanza básica, 83 (66\%) en la enseñanza media y 11 (9\%) tenían algún tipo de estudio superior. La edad promedio de los lactantes fue 5 meses (rango: 1-24 meses), correspondiendo 66 (52\%) a mujeres.

Evaluación de la técnica inhalatoria. El puntaje obtenido por las madres presentó un rango entre 4 y 10 puntos, con una mediana de 9 puntos. La distribución de frecuencias de los puntajes se ilustra en la tabla 2. Dieciséis madres (12,5\%) obtuvieron el puntaje máximo. El porcentaje de cumplimiento de cada paso se ilustra en la tabla 3. Cinco pasos fueron realizados en forma deficiente por más del $10 \%$ de
Tabla 2. Número y porcentaje de pasos realizados correctamente por las madres

\begin{tabular}{crr}
$\begin{array}{c}\text { Número de } \\
\text { pasos correctos }\end{array}$ & Número de madres (\%) \\
\hline 4 & 2 & $(1,6)$ \\
5 & 1 & $(0,8)$ \\
6 & 4 & $(3,1)$ \\
7 & 11 & $(8,6)$ \\
8 & 16 & $(12,5)$ \\
9 & 78 & $(60,9)$ \\
10 & 16 & $(12,5)$ \\
Total & 128 & $(100,0)$ \\
\hline
\end{tabular}

Tabla 3. Porcentaje de cumplimiento según paso evaluado ( $n=128$ madres)

\begin{tabular}{lc}
\hline Acción realizada por la madre & $\begin{array}{c}\% \\
\text { cumplimiento }\end{array}$ \\
\hline Agita el inhalador & 89,4 \\
Inserta el inhalador en la aerocámara & 98,4 \\
Posiciona correctamente al lactante & 97,7 \\
Posiciona correctamente la aerocámara & 99,2 \\
sobre boca y nariz & 99,2 \\
Administra un sólo puff & \\
Espera 10 segundos o 10 respiraciones del & 89,1 \\
lactante & 89,8 \\
Remueve la aerocámara & 17,2 \\
Espera 30-60 segundos antes de repetir & 98,4 \\
el ciclo & 83,6 \\
Agita nuevamente el inhalador & \\
Repite el ciclo completo &
\end{tabular}

madres, correspondientes a los pasos 1, 6, 7, 8 y 10 ; siendo el paso 8 (i.e. espera $30-60$ segundos antes de repetir el ciclo) el de menor cumplimiento, siendo realizado en forma correcta por el $17,2 \%$ de las madres.

\section{Discusión}

El presente estudio demostró que la mayoría de las madres de lactantes hospitalizados por IRA realizaron la TI de manera correcta, presentando un número reducido de errores $(73,4 \%$ de ellas obtuvo puntajes igual o superior a 9 puntos), sin embargo, sólo una de cada ocho 
madres realiza la TI libre de errores u omisiones, cual es la meta a alcanzar por el equipo de salud. Este porcentaje puede considerarse bajo en vista que la mayoría de las madres habían recibido instrucción verbal o demostrativa y tenían experiencia con el uso de inhaladores en episodios previos.

Los resultados de nuestra evaluación concuerdan con la experiencia reportada por Vodoff y cols ${ }^{9}$, quienes evaluaron mediante una escala de 12 ítems a 60 cuidadores de niños asmáticos menores de 4 años, alcanzando solamente 10\% de ellos el puntaje máximo. Por el contrario, la experiencia reportada por Walia y cols ${ }^{2}$ determinó que $72 \%$ de los cuidadores de niños asmáticos menores de 3 años eran "usuarios adecuados” de la TI; sin embargo, la escala de evaluación utilizada fue menos exigente, por cuanto solamente consideró el cumplimiento de 5 ítems considerados “esenciales”. Es importante destacar que ambas experiencias fueron realizadas en niños con diagnóstico de asma bronquial y en escenarios ambulatorios, lo cual no permite establecer comparaciones de manera fácil.

El error más frecuentemente identificado en nuestra evaluación correspondió al tiempo de espera entre el primer y segundo ciclo inhalatorio $(82,8 \%)$. Esta observación concuerda con la serie de Vodoff y cols ${ }^{9}$, en la cual $72 \%$ de los padres no esperaba un lapso de tiempo adecuado entre el primer y segundo ciclo inhalatorio, siendo en esta serie el segundo error más frecuentemente cometido. La principal consecuencia de esta omisión sería impedir un adecuado depósito de fármaco en los pulmones, comprometiendo el resultado de una correcta TI. Otro error frecuente, cometido por una de cada seis madres, fue el omitir la realización de un segundo ciclo inhalatorio, cuya consecuencia podría implicar una reducción mayor al 50\% de la dosis nominal del fármaco administrado en cada instancia inhalatoria. De igual forma, una de cada nueve madres no espera 10 segundos o 10 respiraciones del lactante dentro de la aerocámara tras descargar el inhalador, tiempo considerado como adecuado para permitir al niño vaciar adecuadamente su contenido, teniendo en cuenta que el tiempo de espera óptimo no ha sido internacionalmente estable- cido. Finalmente, una de cada nueve madres no agitó el inhalador previo a la primera inhalación, impidiendo la mezcla homogénea de los compuestos incluidos en el inhalador, los cuales pueden decantar si el inhalador no ha sido utilizado después de un determinado lapso de tiempo ${ }^{10}$.

Al corregir los errores y preguntar las razones que tuvieron las madres para cometerles, se observó que una fuente importante ocurre como consecuencia de una inadecuada entrega de información, muchas veces equivocada, por parte de diferentes agentes de salud (médicos, enfermeras, kinesiólogos y auxiliares, entre otros) al momento de instruir a las madres. Hanania y cols ${ }^{11}$, evaluaron la calidad de la TI realizada por diversos agentes de salud, obteniendo $98 \pm 2 \%$ de acierto (terapeutas respiratorios), $78 \pm 20 \%$ (enfermeras) y $57 \pm 31 \%$ (médicos), reflejando la falta de uniformidad en los conceptos por parte de quienes son responsables de la instrucción y monitorización de los cuidadores y pacientes.

La extrapolación de los resultados de este estudio a la población total de madres que inhalan a lactantes no es del todo precisa, por cuanto los pacientes de esta serie presentan una característica especial, cual es el haber requerido hospitalizarse producto de una IRA. Resulta razonable plantear que las calificaciones de las madres de este estudio deberían ser superiores a las calificaciones que obtendría la población total de madres de lactantes, por cuanto las primeras tuvieron la posibilidad de observar al personal de enfermería realizar la TI en diferentes circunstancias durante su estadía hospitalaria antes de ser evaluadas. Por el contrario, también es razonable plantear que el resultado obtenido por las madres en este estudio podría ser inferior al de la población total de madres de lactantes por el sólo hecho de que sus hijos requirieron hospitalizarse, revelando el fracaso que en su caso tuvo la terapia inhalatoria ambulatoria, condicionado en parte por no haber realizado una TI adecuada. De todos modos, la exploración de estas hipótesis requiere de un diseño de investigación diferente al planteado en nuestro estudio.

En conclusión, el presente estudio identificó los errores más frecuentes cometidos por las 
madres de 128 lactantes hospitalizados por infecciones respiratorias agudas. A pesar de ser una modalidad terapéutica frecuente en nuestro medio, es incorrecto considerar la terapia inhalatoria como un procedimiento sencillo para muchas madres o cuidadores; por el contrario, la educación periódica de los pacientes y sus cuidadores juega un rol insustituible en sus cuidados, por lo que esta tarea debe ser compartida por todos quienes integran el equipo de salud.

\section{Agradecimientos}

Agradecemos a todas las madres que accedieron a participar en este estudio durante la hospitalización de sus hijos.

\section{Referencias}

1.- Fink J, Rubin B: Problems with inhaler use: a call for improved clinical and patient education. Respir Care 2005; 50: 1360-74.

2.- Walia M, Paul L, Satyavani A, Lodha R, Kalaivani M, Kabra SK: Assessment of inhalation technique and determinants of incorrect performance among children with asthma. Pediatr Pulmonol 2006; 41: 1082-7.

3.- Dubus JC: Inhaled treatments delivery in children. Arch Pediatr 2003; 10: 1083-8.

4.- Cole $\mathrm{CH}$ : Special problems in aerosol delivery: neonatal and pediatric considerations. Respir Care 2000; 45: 646-51.

5.- Ortiz B, Pérez FC, Nieto MP, Saiz ML, Crespo P, Aldecoa $C$ : Use of inhaled bronchodilators by primary care patients. Aten Primaria 1996; 18: 497-501.

6.- Lirsac B, Braunstein G: Randomized evaluation of two teaching methods using aerosol dosers. Rev Mal Respir 1991; 8: 559-65.

7.- Horsley MG, Bailie GR: Risk factors for inadequate use of pressurized aerosols inhalers. J Clin Pharm Ther 1988; 13:139-43.

8.- Pedersen S, Frost L, Anfred T: Errors in inhalation technique and efficiency in inhaler use in asthmatic children. Allergy 1986; 41: 118-24.

9.- Vodoff MV, Gilbert B, De Lumley L, Dutau G: Technique d'utilization de la chambre d'inhalation avec masque facial dans l'asthme. Evaluation chez 60 enfants âgés de moins de quatre ans. Arch Pédiatr 2001; 8: 598-603.

10.- Dolovich MB, Fink J: Aerosols and devices. Respir Care Clin N Am 2001; 7: 131-73.

11.- Hanania NA, Wittman R, Kesten S, Chapman KR: Medical personnel's knowledge of and ability to use inhaling devices. Metered-dose inhalers, spacing chambers, and breath-actuated dry powder inhalers. Chest 1994; 105: 111-6. 